\title{
THE QUEST FOR X-RAYS FROM PROTOSTARS
}

\author{
THIERRY MONTMERLE \\ Service d'Astrophysique, Centre d'Etudes de Saclay \\ $C E A / D S M / D A P N I A / S A p$ \\ 91191 Gif-sur-Yvette Cedex, France
}

\section{What is a protostar ?}

The field of low-mass star formation and early evolution has made rapid progress in recent years, thanks in particular to observations in the IR and $\mathrm{mm}$ ranges. The current evolutionary scheme calls for two main stages, themselves divided into two substages (e.g., André \& Montmerle 1994): (i) protostars, comprizing the newly discovered so-called "Class 0 sources", detected mostly or only in the mm range, which are young protostars with estimated ages $\sim 10^{4} \mathrm{yrs}$, and "Class I sources", visible in the near- to mid-IR, which are evolved protostars with estimated ages $\sim 10^{5} \mathrm{yrs}$; (ii) T Tauri stars, which are visible in the IR but also in the optical, the younger being the "classical" T Tauri stars (called "Class II" in the IR), and the "weak-line" T Tauri stars ("Class III" in the IR), with a large age spread of $\sim 10^{6}-10^{7}$ yrs. According to current models (e.g., Shu et al. 1987), protostars consist of a forming star surrounded by an extended envelope (up to $\sim 10,000 \mathrm{AU}$ in radius); the star forms via an accretion disk inside a cavity $\sim$ several $100 \mathrm{AU}$ in radius. The disk probably plays an important role in generating molecular outflows, running through the envelope. Classical T Tauri stars are only surrounded by a disk, which disappears at the weak-line $\mathrm{T}$ Tauri stage.

The important difference between a "young" (i.e., Class 0) protostar and an "evolved" (i.e., Class I) protostar is not so much of structure, but rather of content. In Class 0 protostars the envelope contains most of the (future stellar) mass : $M_{\text {env }} \gg M_{\star}$, while the opposite holds for Class I protostars, where the envelope is comparatively tenuous. Translated into extinction in the visible, a Class I envelope has $A_{V} \sim$ several 10, while for Class $0, A_{V}>100$ or more, i.e., $N_{H} \sim 10^{22}-10^{23}$ $\mathrm{cm}^{-2}$.

\section{Why look for X-rays ?}

As amply demonstrated in the case of $\mathrm{T}$ Tauri stars, and more generally in all late-type stars, including the Sun (as vividly illustrated by the Yohkoh results), $\mathrm{X}$-ray emission is closely linked with magnetic fields, via reconnection of field lines of opposite polarity, while the magnetic fields themselves are believed to originate in the dynamo effect resulting from convective motions (e.g., Montmerle et al. 1993). 
In "ordinary" stars, however, the X-rays come from a hot ( $\left.\gtrsim^{7} \mathrm{~K}\right)$ plasma trapped in magnetic loops anchored to the photosphere. $\mathrm{T}$ Tauri stars and protostars offer more exotic possibilities, with possible combinations of magnetic field lines linking the star, the accretion disk, and the envelope. In T Tauri stars, although the situation likely exists, the comparison between the X-ray properties of classical vs. weak-line $T$ Tauri stars shows no evidence so far for a significant star-disk contribution to the X-ray emission. In protostars, only recently has some $\mathrm{X}$-ray evidence been found for star-disk or star-envelope interactions (see below). The X-rays thus appear as a unique probe of the magnetic structure of very inner regions of protostars. Also, a high $\mathrm{X}$-ray flux may significantly affect the physical state of the circumstellar material, for instance via ionization (accretion, Glassgold et al., 1997, or coupling of matter with the magnetic fields), heating, and also induce various irradiation effects on dust grains, gas chemistry, etc. (e.g., Casanova et al., 1995; Maloney et al., 1996).

There are difficulties, however, because of the high extinction associated with protostars. This extinction results not only of the amount of envelope material along the line-of-sight, but also from the fact that protostars are generally embedded in dense interstellar matter, typically "cores" of molecular clouds, where gas densities exceed $10^{5} \mathrm{~cm}^{-3}$. As a result, reliable estimates of $A_{V}$ are not easy to obtain. Since the X-ray photoelectric cross-section varies roughly as $E_{X}^{-3}$ (e.g., Ryter 1996), X-rays are a priori easier to detect in the higher-energy range for a given sensitivity.

\section{Success of the quest}

In the course of observations of dense molecular clouds, which are the seat of low-mass star formation, and which were already known to harbor X-ray emitting $\mathrm{T}$ Tauri stars, ROSAT and $A S C A$ were able to detect $\mathrm{X}$-ray emission from Class I protostars.

The first detections were obtained on nearby molecular clouds, $\rho$ Oph and $R$ CrA, at distances $\gtrsim 150$ pc from the Sun. In a deep ( $30 \mathrm{ksec})$ ROSAT PSPC exposure, Casanova et al. (1995) found Class I sources in the error boxes (typically $\approx 10-20^{\prime \prime}$ in radius) of X-ray sources in the $\rho$ Oph cloud, but confusion with other IR sources prevented secure identifications. With ASCA, Koyama et al. (1996) detected a cluster of Class I sources in $\mathrm{R} \mathrm{CrA}$, but here again the identifications were not secure, since the typical $A S C A$ error box is $\gtrsim 1^{\prime}$ in radius. A reliable identification could be obtained with the ROSAT HRI, when Grosso et al. (1997) detected a variable X-ray source within $\sim 2^{\prime \prime}$ of an IR source associated with a well-documented Class I source in the $\rho$ Oph cloud, YLW15. More examples are now available, as Table 1 shows.

One way to estimate the efficiency of protostars to convert energy into X-rays is to compute their bolometric-to-X-ray luminosity ratios $L_{X} / L_{b o l}$. As can be seen from Table 1 , most protostars have $L_{X} / L_{b o l} \sim 10^{-3}-10^{-2}$, which is on the high side of the values obtained for $\mathrm{T}$ Tauri stars. The younger protostars, as estimated from their IR and mm properties (see, e.g., André \& Montmerle 1994), tend to have the highest ratios. For these objects, the interpretation of $\mathrm{X}$-ray emission in terms of magnetic activity at the surface of the forming star (especially flares) is fully consistent with that observed on T Tauri stars. 
Table 1 - X-ray detected protostars

\begin{tabular}{ccccccc}
\hline $\begin{array}{c}\text { Source } \\
\text { name }\end{array}$ & Cluster & $\begin{array}{c}\mathbf{A}_{V} \\
{[\mathrm{mag}]}\end{array}$ & $\begin{array}{c}L_{X}\left(10^{30} \mathrm{erg} \mathrm{s}^{-1}\right) \\
(0.1-2.4 \mathrm{keV})\end{array}$ & $\log \left(L_{X} / L_{\text {bol }}\right)$ & Flare ? & Ref. \\
\hline IRS1/TS2.6 & RCrA & 41 & $\sim 1.7$ & -4.3 & No & 1 \\
& & & 2.5 & & No & 2 \\
IRS2/TS13.1 & RCrA & 32 & $\sim 0.9$ & -3.6 & No & 1 \\
IRS5/TS2.4 & RCrA & 39 & $\sim 1.4$ & -3.4 & No & 2 \\
& & & 9.3 & & No & 2 \\
IRS7/R1 & RCrA & 35 & $\sim 6.3$ & -3.4 & Yes & 1 \\
& & & $<1.0$ & -3.4 & Yes & 1 \\
IRS9/R2 & RCrA & 32 & $\sim 1.0$ & & - & 2 \\
\hline YLW15 & $\rho$ Oph & 33 & 160 & -2.1 & No & 3 \\
YLW15 & $\rho$ Oph & 33 & $>10^{3}$ & $>-1.6$ & Yes & 4 \\
& & & $L_{X, t o t}>10^{4}$ & $>1$ & & \\
EL29 & $\rho$ Oph & 57 & 7.3 & -4.4 & Yes & 5 \\
WL6 & $\rho$ Oph & 57 & 4.7 & -3.3 & Periodic & 4 \\
\hline SVS16 & NGC1333 & 28 & 280 & -2 & No & 6 \\
\hline
\end{tabular}

Refs.: (1) Koyama et al. (1996); (2) Neuhaüser \& Preibisch (1997); (3) Casanova et al. (1995); (4) Grósso et al. (1997); (5) Kamata et al. (1997); (6) Preibisch (1997)

$\dagger$ Integrated over all $X$-ray energies.

In one remarkable case, however, the $L_{X} / L_{b o l}$ ratio has been found to be $>$ to $\gg 1$. This value has been reached by YLW15 during a "superflare" (Grosso et al. 1997) lasting a few hours. It cannot be well determined because the value of the extinction, as well as of the temperature (since the HRI has no spectral resolution), is uncertain, but as shown in Table 2 , the X-ray luminosity must have been very high, perhaps as high as $\sim 10^{36} \mathrm{erg} \mathrm{s}^{-1}$. The characteristic cooling time allows to compute the plasma density and the size of the emitting region, as well as the value of the equipartition magnetic field (see Grosso et al. 1997 for details). In the case of YLW15, the plasma density and the magnetic field are more or less solar, but the size of the emitting region (assumed to be in the form of a tube with an aspect ratio $a=$ length/diameter $=10)$ can be of the order of $1 \mathrm{AU}$. The large $L_{X} / L_{b o l}$ ratio, as well as the large size of the emitting region, strongly suggest that the X-ray activity, while still magnetic in origin, cannot be due to the star alone, but rather is the result of star-disk and/or star-envelope interactions (see, e.g., Hayashi et al. 1996). (Another possibility is that the central star is a binary, and then there can be star-star interactions like in the very X-ray-active RS CVn systems.)

\section{Conclusions}

The above results show that large numbers of hard photons are likely to be present in the protostellar systems. Not only are the effects mentioned in Sect. 2 on the circumstellar gas and the dust important, but it is also likely that the disk would be bombarded by shocks and energetic protons associated with the $\mathrm{X}$-ray flares. As the early Sun should have passed through the flaring Class I pro- 
Table 2 - X-ray properties of the YLW15 superflare

\begin{tabular}{llcccc}
\hline & $\mathbf{T}_{X}\left(10^{7} \mathrm{~K}\right)$ & 1 & 3 & 6 & 12 \\
\hline \multirow{2}{*}{$A_{V}=20$} & $\log \mathrm{L}_{X, \text { tot }}^{\S}$ & 33.7 & 34.0 & 34.3 & 34.5 \\
& length (AU) & 0.32 & 0.24 & 0.24 & 0.20 \\
\hline $\mathbf{A}_{V}=\mathbf{3 0}^{\star}$ & $\log \mathrm{L}_{X, t o t}$ & $\mathbf{3 4 . 6}$ & $\mathbf{3 5 . 1}$ & $\mathbf{3 5 . 2}$ & $\mathbf{3 5 . 5}$ \\
& $\operatorname{length}(\mathrm{AU})$ & $\mathbf{0 . 6 4}$ & $\mathbf{0 . 5 2}$ & $\mathbf{0 . 4 8}$ & $\mathbf{0 . 4 4}$ \\
\hline $\mathrm{A}_{V}=40$ & $\log \mathrm{L}_{X, \text { tot }}$ & 35.0 & 35.9 & 36.1 & 36.4 \\
& length (AU) & 1.2 & 1.2 & 0.92 & 0.84 \\
\hline
\end{tabular}

\$ Corrected for extinction. ${ }^{\dagger}$ Length of a magnetically confined plasma tube (aspect ratio $\left.=10\right) .{ }^{\star}$ Best guess for the total visual extinction: $A_{V}=A_{V, I S M}+A_{V \text {,envelope }}$.

tostellar phase, the existence of such shocks, as well as X-ray and particle irradiation phenomena, may resolve several long-standing mysteries (isotopic anomalies, chondrule melting, excess particle irradiation) in ancient meteorites (e.g., Feigelson 1982, Cameron 1995; Lee et al., 1997). Also, the study of protostellar X-rays may hold clues to the origin of outflows, generally thought to be driven by MHD mechanisms.

The next step now is the search for X-rays from the youngest protostars, namely the Class 0 sources, allowing to push the "age barrier" for X-ray emission down to $\sim 10^{4}$ yrs. To do this, an extended energy range, as well as a good sensitivity, are required to overcome the large extinction of these objects. Future $\mathrm{X}$-ray satellites, $A X A F, X M M$ and $A s t r o-E$, have the required characteristics. In addition, their spectroscopic capabilities should allow to directly measure the extinction, leading to more accurate determinations of the $\mathrm{X}$-ray luminosities. If $\mathrm{X}$-rays are indeed found from these very young stars, then this will show that $\mathrm{X}$-rays are a lifetime companion to all stages of stellar evolution.

\section{References}

André, P., \& Montmerle, T., 1994, Ap.J., 420, 837

Cameron, A.G.W. 1995, Meteoritics, 30, 133

Casanova, S., Montmerle, T., Feigelson, E.D. \& André, P. 1995, Ap.J., 439, 752

Feigelson, E.D. 1982, Icarus, 51, 155

Glassgold, A.E., Najita, J., \& Igea, J. 1997, Ap.J., 480, 344

Grosso, N., Montmerle, T., Feigelson, E.D., André, P., Casanova, S., \& GregorioHetem, J. 1997, Nature, 387, 56

Hayashi, M.R., Shibata, K., \& Matsumoto, R. 1996, Ap.J., 468, L37

Kamata, Y., et al., PASJ, in prèss

Koyama, K., Hamaguchi, K., Ueno, S., Kobayashi, N. \& Feigelson, E.D. 1996, PASJ, 48, L87

Lee, T., Shu, F.H., Shang, H. \& Glassgold, A.E. 1997, Science, in press

Maloney, P.R., Hollenbach, D.J., \& Tielens, A.G.G.M. 1996, Ap.J., 466, 561

Montmerle, T., Feigelson, E.D., Bouvier, J., \& André, P. 1993, in Protostars \& Planets III, U. of Arizona Press, p. 689

Neuhäuser, R. \& Preibisch, Th. 1997, Astr.Ap., 322, L37

Preibisch, T. 1997, Astr.Ap., 324, 690

Ryter, C.E. 1996, Astr.Sp.Sci., 236, 285

Shu, F.H., Adams, F.C., \& Lizano, S. 1987, Ann.Rev.Astr.Ap., 25, 23 